\title{
Les zones de protection du patrimoine architectural, urbain et paysager (ZPPAUP), une forme de gouvernance patrimoniale? Mathieu GIGOT ${ }^{1}$ \\ UNIVERSITE FRANÇOIS-RABELAIS, TOURS
}

\section{Résumé}

Dans un contexte global où la prise en compte du patrimoine s'intensifie tant au niveau de la diversité des espaces traités que de leur surface, de multiples mesures de protection et de sauvegarde de ces éléments patrimoniaux existent. En France, c'est l'Etat qui reste compétent dans ce domaine malgré les lois de décentralisation des années 1980 donnant aux diverses collectivités territoriales une certaine autonomie. Cependant, une procédure échappe partiellement à la règle et introduit une nouvelle façon de penser l'action patrimoniale en privilégiant la négociation et le consensus: dans la lignée des politiques contractuelles liées à la décentralisation, les zones de protection du patrimoine architectural, urbain et paysager (ZPPAUP) permettent en effet une mise en débat de la patrimonialisation sur une scène locale de négociation en faisant plus amplement intervenir la collectivité locale concernée. Ce mode d'action s'apparente à un processus de gouvernance de l'action publique mais sans aller jusqu'au bout de la démarche, pour ne constituer qu'un mode de régulation spécifique de l'action patrimoniale locale. A partir de l'exemple d'une ville française, Orléans, cet article interroge la pertinence de l'utilisation des notions de gouvernance et de régulation patrimoniale dans la mise en place récente d'une zone de protection du patrimoine architectural, urbain et paysager sur un centre ancien.

\section{Abstract}

ZPPAUP : a kind of patrimonial governance?

In a global context where the consideration of heritage is growing about a variety of the concerned spaces and their surfaces, there are many protective measures for their patrimonial items. In France, the State is competent in this domain in spite of the decentralisation laws (1980') which give a kind of autonomy to local authorities. However, a procedure escapes from the rule and introduces an another way to think patrimonial action by emphasizing negociation and consensus : in succession of contractual policies linked to decentralisation, architectural, urban and landscape heritage protection areas (ZPPAUP) enable debates on the local scene of negociation giving the local authorities a largest space for intervention. This kind of action is similar to the governance process but is abortive and constitutes a specific kind of patrimonial regulation of local action. In the case of a French city, Orléans, this contribution questions relevance of such notions as governance and patrimonial regulation about the recent implementation of ZAPPUP in historical city centre.

\footnotetext{
${ }^{1}$ UMR 6173 CITERES, équipe CoST
} 


\section{INTRODUCTION}

En France comme dans la plupart des pays développés, la préservation du patrimoine connaît un engouement extraordinaire: "dans un monde où la modernité d'abord puis la globalisation ont développé la crainte de l'homogénéisation et de la banalisation, sa valeur symbolique s'est considérablement renforcée" (TOMAS, 2004). Une telle conception du monde amène cet objet, construit socialement, au cœur des politiques publiques si bien que les centres urbains, s'ils ne sont pas muséifiés, sont tout du moins largement préservés. Si la conservation d'éléments du passé n'est pas récente (les premières mesures conservatrices françaises étant datées de la Révolution de 1789, comme le montre Françoise CHOAY (1996)), la préservation et la restauration de quartiers entiers sont un phénomène plus récent.

La grande loi française en matière de protection du patrimoine date du 31 décembre 1913 et concerne le classement des monuments historiques ${ }^{2}$. Il s'agit bien d'opérations ponctuelles de sauvegarde: la conception patrimoniale qui prévaut étant bien celle d'un patrimoine monumental. En 1943, l'Etat met en place un système de protection de l'environnement proche des monuments historiques classés ${ }^{3}$ (passant d'une protection ponctuelle à une protection plus zonale des espaces), tout en conservant la conception de la monumentalité patrimoniale puisque les abords des monuments historiques ainsi protégés le sont pour assurer un cadre paysager cohérent par rapport au monument historique classé. Ces mesures s'étoffent une vingtaine d'années plus tard avec la création en 1962 des secteurs sauvegardés ${ }^{4}$, véritables documents d'urbanisme ayant pour objet la préservation de quartiers historiques entiers. L'accent n'était dès lors plus mis sur un monument classé pour lequel étaient mis en place des périmètres de protection, mais bien sur chacun des immeubles qui constituaient les quartiers anciens. Cette procédure constitue un point de rupture dans la définition du patrimoine car un glissement s'opère d'un patrimoine monumental vers un patrimoine urbain, autrement dit "le petit patrimoine". Si une telle conception du patrimoine n'était pas nouvelle (John RUSKIN l'évoquait déjà au $\mathrm{XIX}^{\mathrm{e}}$ siècle), jamais une loi n'avait permis la protection réelle de tels ensembles urbains pour leurs valeurs propres.

Aussi efficaces qu'ils soient, les secteurs sauvegardés restent des documents très centralisés, dans la continuité de la vision d'un Etat tout puissant. Une liste de 400 villes françaises pouvant prétendre à la création d'un secteur sauvegardé avait été établie dès le lancement de la procédure, mais seuls 92 ont été créés. Depuis, le contexte institutionnel Français a considérablement évolué et la France s'est fortement décentralisée. Ces lois de décentralisation ${ }^{5}$ ont depuis une vingtaine d'années introduit de nouveaux rapports entre les collectivités territoriales et de facto entre l'Etat et ces collectivités, faisant entrer dans l'élaboration des politiques publiques locales l'idée de coopération, de

\footnotetext{
${ }^{2}$ Un monument classé jouit d'une forte protection imposée par l'Etat aux propriétaires concernés, qu'ils soient publics ou privés. Le classement d'un monument implique un droit de regard de l'Etat sur l'entretien et les divers travaux à mener

${ }^{3}$ Loi ${ }^{\circ} 92$ du 25 février 1943, concernant la protection des abords des monuments historiques

${ }^{4}$ Loi Malraux du 4 août 1962 (articles L.313-1 à L.313-3 \& R.313-1 à R.313-29 du Code de l'urbanisme)

${ }^{5}$ Loi Defferre du 2 mars 1982 relative aux droits et libertés des communes, des départements et des régions
} 
négociation et plus récemment en France le concept de gouvernance. Dans le domaine du patrimoine, la première vague de décentralisation n'a pas provoqué de changements radicaux pour les collectivités territoriales mis à part la possibilité de créer des zones de protection du patrimoine architectural et urbain (et paysager par la suite $)^{6}$, les ZPPAU. Cette procédure, que certains assimilent à des "mini " secteurs sauvegardés, mais décentralisés, a connu un certain succès puisque l'on n'en dénombrait pas moins de 420 en 2003. En 2004, une deuxième vague de décentralisation patrimoniale a été initiée en France, mais elle n'a consisté qu'à céder aux collectivités locales certains monuments nationaux, ce qui reste une avancée mineure au regard d'autres domaines de compétences plus fortement décentralisés. La gestion même des éléments patrimoniaux en particulier et des espaces urbains protégés (au titre de la loi de 1943 ou d'un secteur sauvegardé) restent de la compétence de l'Etat car le patrimoine est le bien commun de la nation. Même dans le cas des ZPPAUP (le dernier " $\mathrm{P}$ » de paysage ayant été ajouté en 1993), la décentralisation reste limitée. Si cette procédure introduit bien l'idée d'une horizontalité des relations entre acteurs publics (donc une certaine forme d'égalité d'action entre eux) et, de fait, l'amorce d'une gouvernance patrimoniale, n'est-il pas illusoire de penser que les pratiques suivent ce discours? Ces ZPPAUP qui dans les propos laissent une part belle aux collectivités locales dans la gestion du patrimoine, sont-elles véritablement des procédures décentralisées, au sens où l'autonomie de la collectivité prime sur l'action étatique?

Cet article tend à interroger l'existence et le fonctionnement d'une gouvernance patrimoniale introduite par les ZPPAUP. Je prendrai à titre d'exemple le montage de la ZPPAUP d'Orléans, qui à l'instar de ses voisines cherche à valoriser son patrimoine urbain.

\section{1 : ORLEANS : UNE VILLE EN QUETE DE RECONNAISSANCE}

\section{1 : Situation}

Orléans est une ville de taille moyenne (environ 116000 habitants), capitale de la Région Centre qui jouit et souffre en même temps de sa proximité avec Paris (130 kilomètres). Au fil du temps, l'image de cette ville a profondément évolué pour perdre peu à peu une importance qu'elle avait auparavant. La brochure d'information présentant les grands projets urbains de l'agglomération est assez évocatrice lorsqu'elle stipule en préambule: "Orléans! Ville pétrie d'histoire; souvent glorieuse, parfois assoupie »7. En effet, entre Jeanne d'Arc, figure omniprésente dans la ville, et les vinaigreries qui assurèrent il fut un temps la prospérité économique de la ville mais qui ne laissent aujourd'hui que des friches à reconvertir, l'on décèle bien l'importance politique ou économique de cette cité qui aujourd'hui semble souffrir d'une mauvaise presse, comme le remarquait Michel GRESILLON (1995) : "Orléans a connu une grande rupture dans son histoire, dont les stigmates sont aujourd'hui encore dans le défaut

\footnotetext{
${ }^{6}$ Loi no $83-8$ du 7 janvier 1983 relative à la répartition de compétences entre les communes, les départements, les régions et l'Etat (art. 69 à 72)

${ }^{7}$ Extrait de «Orléans 2015 : promenons-nous demain »
} 
d'identité et d'image, ainsi que dans les mentalités qu'elle se donne ». En terme d'image, élément ô combien essentiel dans les politiques de valorisation des villes à l'heure actuelle, elle ne parvient pas à se positionner. Si sa rivale ligérienne elle aussi, Tours, mise sa politique de communication sur le cadre de vie exceptionnel dont elle dispose grâce à un environnement inscrit au patrimoine mondial de l'UNESCO (c'est en réalité tout le val de Loire qui est inscrit), un secteur sauvegardé ancien et donc un patrimoine mis en valeur de longue date et une proximité temporelle avec la capitale (55 minutes en TGV) ; Orléans ne parvient pas encore à exploiter le filon patrimonial, non par manque d'éléments patrimoniaux (on dénombre pas moins d'une soixantaine de monuments historiques sur la commune et la ville est aussi incluse dans l'espace ligérien inscrit au patrimoine mondial de l'UNESCO), mais par défaut de document protecteur et de connaissance du patrimoine orléanais.

\section{2 : Un projet urbain pour la ville}

Loin de rester inerte, l'équipe municipale actuelle a élaboré pour Orléans et son agglomération une multitude de projets urbains, architecturaux, etc. Ainsi, la municipalité a dressé une liste de ces projets dans la brochure précitée "Orléans 2015: promenons-nous demain" (Le Document 1 ci-après montre les projets dans la zone du centre-ville). Il s'agit avant tout d'améliorer sensiblement l'image de la ville pour la rendre attractive et y développer le tourisme. Certains acteurs municipaux déplorent le fait qu'Orléans soit sur la route des mondialement connus " châteaux de la Loire (ou plutôt que la ville " ne soit que " sur le chemin). Ainsi, les touristes passent mais ne s'arrêtent pas... Sans porter préjudice à la fréquentation touristique de la vallée de la Loire qui jouit d'une solide réputation, la Ville voudrait réussir à mettre en valeur ses atouts, au nombre desquels figure son patrimoine, afin de retenir les touristes.

C'est donc bien dans cette optique que la municipalité s'est penchée sur la protection mais bien plus sur la valorisation de son patrimoine architectural et urbain, par la mise en place d'une procédure de sauvegarde du patrimoine jusqu'alors inexistante. C'est d'ailleurs une curiosité comme l'énonçait l'architecte des bâtiments de France qui considérait à juste titre qu'Orléans aurait dû jouir, comme ses semblables (Tours ou Bourges par exemple) d'un secteur sauvegardé. Mais pour des raisons politiques, ce dernier n'a pu se faire, laissant ainsi à cette capitale un patrimoine méconnu qu'elle ne commence seulement qu'à redécouvrir. Les différents acteurs en lisse dans la protection patrimoniale ont opté pour la mise en place d'une ZPPAUP qui, plus encore que de simplement s'intégrer au projet urbain, semble en constituer la base. Il ne s'agit pas de protéger le patrimoine uniquement pour sa valeur historique et mémorielle, puisque cet outil, comme ses semblables (en particulier les secteurs sauvegardés), est aussi un instrument de connaissance, un outil pédagogique, permettant l'obtention d'une certaine reconnaissance (car créer un outil de protection du patrimoine présuppose que ce patrimoine existe) et l'octroi de labels, tel le label 
des villes et pays d'art et d'histoire ${ }^{8}$, gage d'une qualité architecturale d'une ville et véritable outil de communication.

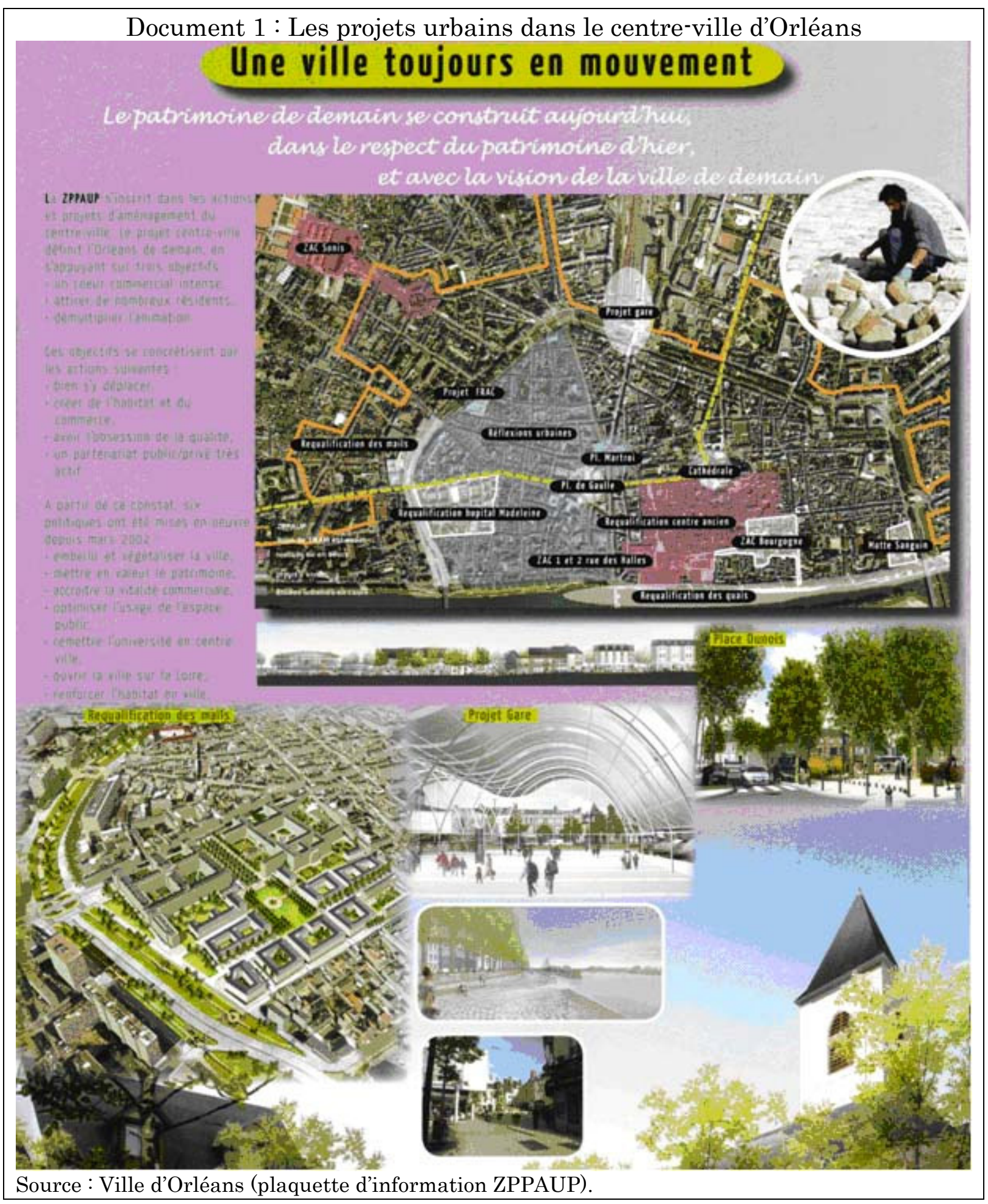

\footnotetext{
${ }^{8}$ Le label «Villes et pays d'art et d'histoire » est attribué par le ministère de la Culture et de la Communication à des collectivités locales qui souhaitent valoriser leur patrimoine, favoriser la création architecturale et promouvoir la qualité dans les espaces bâtis ou aménagés. Cette volonté se traduit par la mise en œuvre d'une convention "Ville et pays d'art et d'histoire», élaborée en concertation avec les communes. Elle définit des objectifs précis et comporte un volet financier
} 


\section{2: LES ZPPAUP : DES OUTILS «DECENTRALISES》 DE PROTECTION DU PATRIMOINE}

Les ZPPAUP sont nées en même temps que l'idée d'une décentralisation des compétences de l'Etat vers les communes. Si le volet paysager n'existait pas à la base (ce n'est qu'en 1993 que sera rajouté le dernier P du sigle), l'accent fut mis nettement sur la préservation du patrimoine avec deux idées fortes:

- L'objectif principal de ces documents est bien de laisser l'initiative de leur mise en place à la commune, même si l'élaboration se fait sous forme de partenariat avec les services de l'Etat;

- D'autre part, il s'agissait de simplifier la gestion du patrimoine bâti en créant un zonage patrimonial suspendant les périmètres de protection des monuments historiques de la loi des abords de $1943^{9}$.

\section{1 : Des documents locaux de protection du patrimoine}

Le code du Patrimoine énonce dans son article L.642-1 que "sur proposition ou après accord du conseil municipal des communes intéressées, des zones de protection du patrimoine architectural, urbain et paysager peuvent être instituées autour des monuments historiques et dans les quartiers, sites et espaces à protéger ou à mettre en valeur pour des motifs d'ordre esthétique, historique ou culturel ». Ainsi formulée, cette définition des ZPPAUP montre la diversité des patrimoines potentiellement éligibles au statut de "patrimoine à protéger " d'une part mais aussi l'importance de la volonté locale de créer ces types de procédures.

Contrairement aux procédures classiques de protection du patrimoine urbain (abords, secteurs sauvegardés et sites ${ }^{10}$ ), les ZPPAUP intègrent véritablement la dynamique de l'action publique locale en ce sens que l'initiative de leur création peut (et devrait) venir des conseils municipaux et que leur mise en place ne peut se faire qu'avec leur accord. Cependant, la loi dit aussi que les ZPPAUP peuvent être créées après avis du conseil municipal, sans qu'il en ait fait la proposition. Ce ne sont donc pas toujours les communes qui ont brusquement l'idée de mettre en place ce type de procédure, inconnue ou trop lourde à leurs yeux. Bien souvent, et c'est ce qu'il faut comprendre implicitement dans la formulation du

\footnotetext{
${ }^{9}$ La loi du 25 février 1943 sur les abords des monuments historiques stipule qu'un périmètre de protection de 500 mètres de rayon est établi autour de chaque monument historique classé. Dans ce périmètre, les permis de construire ainsi que les autorisations de travaux sont soumis à l'avis de l'architecte des bâtiments de France, représentant de l'Etat en matière de patrimoine à l'échelon départemental. Ce dernier donne un avis simple (c'est-à-dire non obligatoire) ou conforme (obligatoire) suivant si le critère de co-visibilté de la construction envisagée avec l'édifice protégé est avéré ou non. On parle de co-visibilité ou de "champ de visibilité » lorsqu'un édifice est au moins en partie dans les abords d'un monument historique et visible depuis lui ou en même temps que lui.

Selon la loi du 25 février 1943, est considéré dans le champ de visibilité d'un immeuble protégé «tout autre immeuble nu ou bâti, visible du premier ou visible en même temps que lui et situé dans un périmètre n'excédant pas 500 mètres »

${ }^{10}$ La loi du 2 mai 1930 relative à la protection des monuments naturels et des sites de caractère artistique, historique, scientifique, légendaire ou pittoresque permet l'inscription ou le classement de sites naturels ou bâtis. L'inscription d'un site est une procédure étatique qui, en milieu urbain, se justifie par la nécessité de protéger une zone réduite mais intéressante du point de vue architectural
} 
code du patrimoine, ce sont les architectes des bâtiments de France qui proposent la mise à l'étude d'une ZPPAUP, que la commune accepte ou refuse selon son programme d'action. Dès lors, il convient de réfléchir sur les possibilités de mise en oeuvre de ces documents dans chaque contexte local d'action. En effet, la possibilité de création d'une telle procédure se trouve pour partie conditionnée par les types de rapports entretenus avant la formulation de la proposition, entre la commune et les services de l'Etat, et principalement l'architecte des bâtiments de France, acteur central de la protection du patrimoine dans les centres urbains.

\section{2 : Simplification de la gestion patrimoniale et détachement de l'Etat}

En plus de la nouvelle liberté de décision laissée aux collectivités, les ZPPAUP permettent aussi de simplifier la gestion du patrimoine. Un des aspects importants de ces procédures est bien la grande diversité des espaces qu'elles peuvent traiter. Contrairement aux secteurs sauvegardés, exclusivement réservés aux centres anciens, les ZPPAUP peuvent être créées autant dans des quartiers centraux que dans des zones plus péricentrales voire même rurales (d'où l'importance du volet paysager de 1993).

Si les espaces traités sont divers, les modes de protection à l'intérieur des ZPPAUP restent équivalents, de zone en zone. Le principal atout de ces procédures, qui sans doute fonde leur succès certain, c'est qu'une fois mise en place, la zone se substitue aux périmètres de protection des monuments historiques, vastes espaces dans lesquels l'architecte des bâtiments de France a un pouvoir important, souvent gênant pour les collectivités. Dans le cas d'Orléans, la ZPPAUP suspend beaucoup de périmètres de protection (voir Document 2), retirant ainsi à l'architecte des bâtiments de France la gestion d'une zone importante dont la collectivité locale sera pour partie gestionnaire. C'est donc une première réponse aux problèmes de la loi des abords des monuments historiques, avant la possibilité de créer des périmètres de protection modifiés depuis la loi solidarité et renouvellement urbain de 2000 (adaptation du périmètre en fonction des caractéristiques de l'environnement proche du monument classé).

La loi des abords de 1943 est très critiquée pour deux raisons essentielles :

- D'une part, les cercles de 500 mètres de rayon sont considérés comme arbitraires puisqu'ils sont automatiquement créés dès lors qu'un édifice est classé monument historique. Leur emprise spatiale ne fait pas toujours sens : dans certains cas, 500 mètres ne suffisent pas à protéger l'environnement du monument considéré et dans d'autres cas, 50 mètres pourraient suffire. De plus, les espaces ainsi protégés ne le sont pas pour eux-mêmes mais bien par rapport au monument historique générant le périmètre de protection. Il n'y a donc ici aucune reconnaissance de l'intérêt patrimonial d'ensembles urbains même si des contraintes pèsent sur ces espaces : ces "ronds bêtes et méchants" (MORAND-DEVILLER, 1985) ne sauraient créer un véritable zonage patrimonial, porteur d'une identité et d'une reconnaissance historique des espaces. 
- D'autre part, à une échelle plus institutionnelle, la gestion des ces espaces est critiquée puisque seuls les architectes des bâtiments de France ont une forme de pouvoir dans ces espaces. Leurs avis, lorsqu'ils sont conformes, donc obligatoires, s'imposent aux collectivités qui ne peuvent les discuter. De ce fait naissent de nombreux conflits entre les collectivités et l'architecte des bâtiments de France. On comprend dès lors que la réalisation des projets urbains dans les centres anciens soumis à la loi des abords ne peut se réaliser que si les relations entre la municipalité concernée et l'architecte des bâtiments de France, qui a un pouvoir de veto important et qu'il est seul à détenir, se passent bien. Les ZPPAUP permettraient d'amoindrir ce "privilège exorbitant" (MIRIEU DE LABARRE, 2004) des architectes des bâtiments de France.

L'intérêt des ZPPAUP est donc double: d'une part, elles suspendent les périmètres de protection des monuments historiques et permettent donc de négocier un zonage de protection cohérent; d'autre part, la décision de les mettre en place est d'échelle communale, voire intercommunale, même si la création se fait par arrêté préfectoral et même si l'élaboration se fait en concertation avec les services de l'Etat.

Le zonage de la ZPPAUP d'Orléans a ainsi dû être négocié, en particulier en ce qui concerne les faubourgs. La ville s'organise avec un centre "intra-mail " et une multitude de faubourgs qui ont fleuri autour des grandes voies de communication desservant le centre-ville. Le patrimoine dans ces faubourgs est tout aussi important que celui du centre-ville, bien que plus récent et c'est bien sur ce point que la négociation fut de mise puisque cela étendait la zone au risque d'enrayer certains projets d'aménagement. En même temps, cette extension a permis de suspendre un certain nombre de périmètres de protection, considérés comme inutiles, comme le montre le Document 2 , permettant à la ville de ne plus se référer à l'architecte des bâtiments de France au sein de la vaste zone ainsi libérée de " contraintes patrimoniales".

C'est précisément cette forme de mise en œuvre de l'outil qui suscite un intérêt chez les chercheurs: la teneur même de la procédure implique des discussions entre les collectivités et les services de l'Etat. C'est une nouvelle forme de régulation de l'action patrimoniale qui se met en place impliquant des processus de négociation, de mise en débat de l'action publique. 


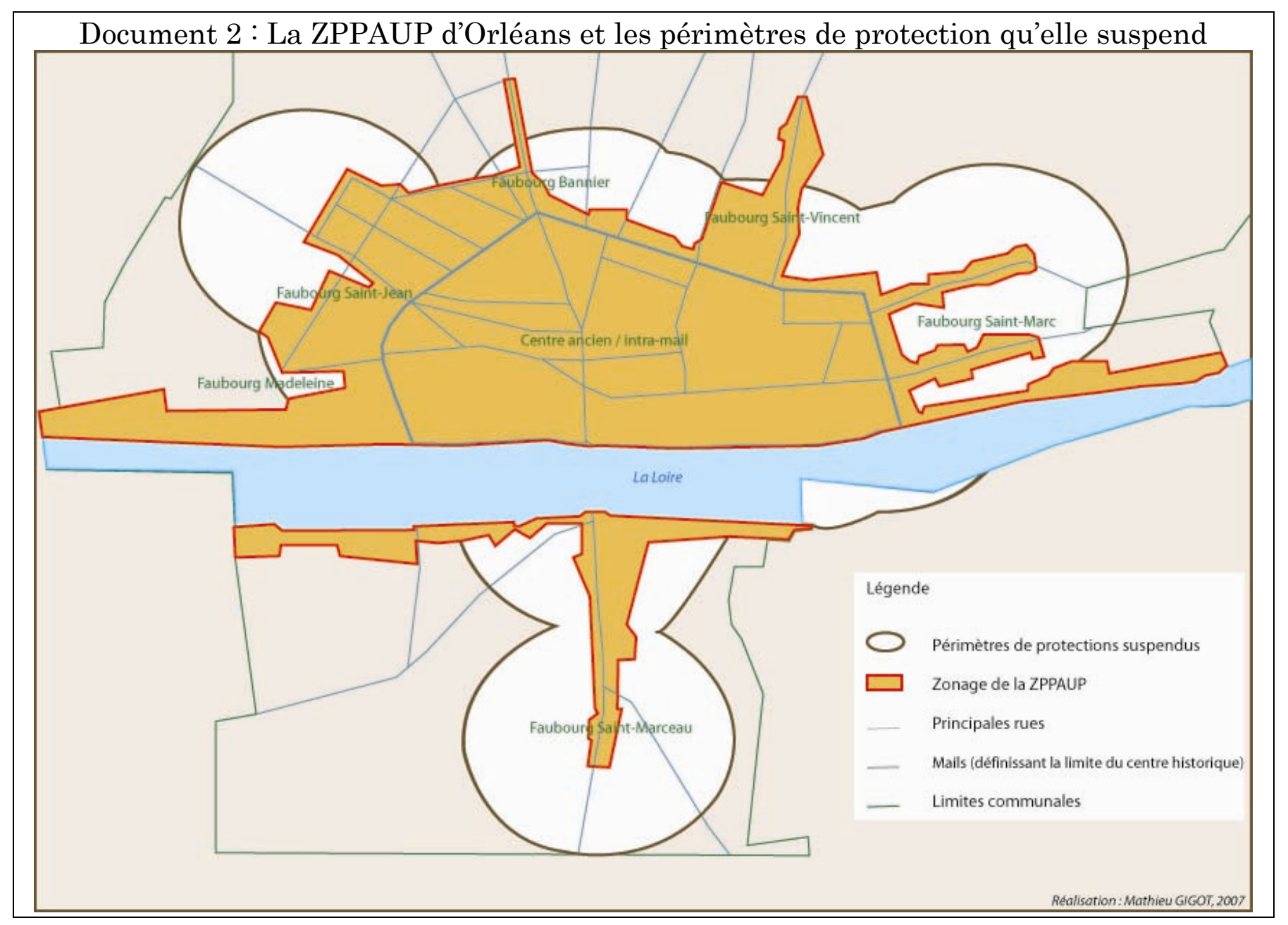




\section{3 : GOUVERNANCE PATRIMONIALE OU REGULATION ?}

\section{1 : La ZPPAUP, une procédure contractuelle}

Les ZPPAUP peuvent aisément s'apparenter à des contrats, non pas au sens juridique du terme, mais bien au sens commun, dans la mesure où un accord est accepté par les parties prenantes, Etat et collectivité principalement.

La notion de contrat, qui rejoint celle de partenariat, s'est développée en même temps que se tramait la décentralisation : Jean-Pierre GAUDIN (1997) précise à juste titre que " bien que l'un ne soit pas la conséquence mécanique de l'autre, le développement des politiques contractuelles ne peut être compris indépendamment du contexte de décentralisation en France ". En effet, la multiplication des collectivités territoriales autonomes, l'importance croissante de leur pouvoir et la nécessité d'un dialogue entre elles a conduit à une contractualisation des politiques publiques.

Les ZPPAUP sont construites sur cette base de partenariat. Il s'agit véritablement d'un contrat entre la collectivité d'une part et l'Etat via l'architecte des bâtiments de France d'autre part. Bien sûr, une multitude d'acteurs interviennent dans le champ patrimonial mais les grandes décisions restent aux mains des élus et du service départemental de l'architecture et du patrimoine. En cela, la mise en place des ZPPAUP définit un nouveau cadre d'action, en instituant une nouvelle forme de régulation du jeu des acteurs patrimoniaux, voire une sorte de gouvernance de l'action patrimoniale.

\section{2 : Une forme de gouvernance de l'action publique?}

Le terme de gouvernance semble approprié pour décrire le développement des procédures contractuelles entre les collectivités territoriales mais aussi la participation grandissante (du moins dans les discours) des citoyens: Catherine BARON (2003) énonce que "c'est bien la problématique du passage d'une structure hiérarchique du pouvoir à une structure de type réseaux qui a justifié le recours à un nouveau terme, celui de gouvernance». Dans les politiques patrimoniales cependant, la participation citoyenne semble moindre, contrairement aux politiques de développement durable par exemple, qui souvent s'appuient sur une forte concertation et un affichage très clair de la politique envisagée. A Orléans, la communication autour du document fut à ce point timide que l'enquête publique fut désertée par les habitants. La gouvernance, si tant est qu'elle existe, ne consistait qu'en un jeu entre différents acteurs publics, les associations ou les habitants n'étant pas ou peu impliqués dans la mise en place de la procédure. Seules des informations destinées à les sensibiliser au problème patrimonial filtraient, mais rien de précis concernant le fond du document. Ainsi, les ZPPAUP peuvent s'afficher comme des documents pédagogiques extrêmement intéressants compte tenu des études qu'ils nécessitent, mais bien souvent, l'enjeu essentiel de ces procédures est oublié : l'explication d'une prise d'autonomie de la collectivité locale, certes relative, n'est pas développée bien que ce soit une démarche très importante et une avancée non négligeable dans l'histoire des relations entre la commune et les services départementaux de l'architecture et du 
patrimoine. Cependant, même en ne considérant que les acteurs publics, il n'est pas certain que l'on puisse qualifier l'action publique comme issue d'une gouvernance puisque si le fonctionnement en réseau existe bien, il reste chapoté par l'Etat, garant de la sauvegarde du patrimoine: "si l'Etat central n'impose plus de façon directe la définition des problèmes à traiter, il se réserve néanmoins une position d'arbitrage, fixe les règles du jeu et identifie les acteurs légitimes. Il garde ainsi, de manière indirecte, un pouvoir relativement important " (HERNANDEZ, 2003). Ceci est vrai pour la plupart des documents d'urbanisme mais revêt une importance beaucoup plus marquée au niveau du patrimoine. Bénédicte DELAUNAY (1995) exprime cette idée à travers cette phrase concernant la procédure de création des ZPPAUP : "En toute hypothèse, c'est une autorité de l'Etat qui prend la décision finale, ce qui permet à ce dernier de conserver la responsabilité de la sauvegarde du patrimoine ». La gouvernance patrimoniale ne paraît plus être qu'un discours sans véritables applications concrètes: si le terme est plaisant, sa mise en œuvre complète semble moins réalisable.

\section{4: LES ZPPAUP, DES NOUVELLES FORMES DE REGULATION PATRIMONIALE}

\section{1 : La régulation plutôt que la gouvernance}

Désormais, le terme de régulation ne s'applique plus qu'à une forme de contrôle de l'Etat sur les marchés : il est aussi utilisé pour décrire les formes de stabilisation des rapports entre les acteurs publics dans les politiques publiques: " la régulation est ici une façon de décrire le mode de prise de décision qui domine dans un système politique" (WOLL, 2004). Dans ce cadre, les ZPPAUP peuvent être considérées comme des instruments de régulation de l'action patrimoniale dans la mesure où elles constituent un cadre de référence stable, élaboré de manière négociée. Dans la mise en place des ZPPAUP en général et en particulier dans le cas d'Orléans, nous parlerons beaucoup plus volontiers d'une régulation concertée que d'une gouvernance patrimoniale, tant le terme présuppose une interrelation des acteurs sous couvert de partenariat. Or les enjeux de la patrimonialisation semblent tels que l'Etat garde toujours une part de responsabilité importante, ne laissant aux communes que l'impulsion d'une politique patrimoniale et éventuellement une part d'appréciation soumise à négociation. Si la commune peut refuser la création des ZPPAUP, elle ne peut qu'accepter de négocier avec l'Etat, sous peine de ne jamais voir aboutir la procédure. Si le partenariat existe bien, il reste forcé par la loi, comme un passage obligé. Certes, ces partenariats peuvent tout à fait être sincères comme à Orléans où les acteurs, étatiques ou municipaux, semblent bien avoir travaillé main dans la main (GIGOT, 2007). Mais les jeux de pouvoirs, édictés par la procédure en elle-même, biaisent considérablement, à mon sens, l'idée d'une véritable gouvernance patrimoniale, d'autant plus que si les acteurs institutionnels négocient, les citoyens se trouvent quasiment exclus du processus (du moins à Orléans). 
C'est bien le problème du concept de gouvernance que d'être un peu "fourre tout»: le terme s'utilise facilement lorsqu'il s'agit de décrire une organisation du pouvoir sous forme de partenariat, "c'est un moyen pour dire que ce qui se passe actuellement n'est pas identique à ce qui avait lieu hier» (LORRAIN, 1998). Il y a là une sorte de facilité, un tiroir sans clef que les chercheurs peuvent ouvrir sans peine. Mais à y regarder de plus près, il semblerait que le concept ne soit pas apte à décrire toutes les situations de partenariats entre acteurs publics et / ou privés. Dans le cas orléanais, il apparaît que l'outil de gouvernance ne soit pas le bon pour évoquer justement ce qui se passe réellement puisque le jeu de négociation est biaisé par la règle même de ce jeu.

\section{2 : Une « nouvelle » régulation patrimoniale ?}

Si les ZPPAUP permettent de contractualiser l'action patrimoniale et de définir un cadre d'action stable dans le temps (ce sont bien des documents à vocation pérenne), il n'est pas certain que le vocabulaire décrivant comme " nouvelles" les formes de relations entre les acteurs soit approprié. En effet, la régulation patrimoniale ainsi définie n'est pas toujours si neuve qu'elle le prétend. Dans beaucoup de cas, la procédure de ZPPAUP ne fait que formaliser et expliciter des rapports préexistants entre l'Etat et la collectivité.

Nous avons bien spécifié que dans les centres anciens, l'architecte des bâtiments de France intervient au nom de la protection des abords des monuments historiques, souvent fréquents dans les centres-villes français. Cela sous tend bien que la commune, qui instruit les permis de construire après avoir élaboré un plan d'occupation des sols ou un plan local d'urbanisme, entretient de fait des rapports avec l'architecte des bâtiments de France qui doit rendre ses avis conformes. C'est cette relation préétablie qui va bien souvent dicter la bonne marche des ZPPAUP. Si les élus et l'architecte des bâtiments de France s'entendent et que leurs avis ne divergent pas totalement, alors une ZPPAUP est envisageable. A contrario, il y a fort peu de chance que sa mise en place soit rendue possible lorsque ces deux acteurs sont d'ors et déjà en conflit. C'est bien ce que j'ai pu observer à Orléans, où l'adjoint à l'urbanisme et l'architecte des bâtiments de France du Loiret s'entendait fort bien avant même que l'idée de ZPPAUP ne soit lancée. C'est d'ailleurs grâce à ces rapports de partenariat que l'architecte des bâtiments de France a proposé la mise en place de ce type de procédure. Et tous les acteurs de préciser que l'entente et la coopération préalable des services de la Ville et du service départemental de l'architecture et du patrimoine furent des éléments indispensables à la création d'une ZPPAUP.

En ce sens, le terme de "nouvelle" régulation patrimoniale n'est pas strictement approprié puisque l'on peut y voir une simple formalisation d'une régulation déjà appliquée mais de manière fermée. Cependant, si l'on retient la définition de Patrick LE GALES (1998) qui énonce que «l'on peut parler de régulation lorsque l'on peut, par exemple, mettre en évidence des relations relativement stabilisées entre des acteurs, des groupes sociaux, qui permettent la répartition de ressources selon les normes et règles explicites ou implicites ", il semblerait que dans certains cas l'on puisse énoncer le terme de renouveau. Tout 
dépend des relations qu'entretiennent les acteurs avant la mise en place de la procédure. A Orléans par exemple, le terme de nouvelle régulation n'est pas approprié puisque la ZPPAUP a surtout permis d'expliciter des relations déjà vécues.

C'est donc tout l'intérêt de la procédure de ZPPAUP que d'expliciter et de rendre lisible cette régulation. Ce point de vue reste toutefois à nuancer car la mise en place d'une ZPPAUP implique l'ouverture d'une scène locale de négociation. C'est un moment privilégié de l'action publique qui met autour de la table les acteurs du champ patrimonial. Tous discutent et défendent des intérêts qui leurs sont propres et ils doivent arriver à trouver un compromis acceptable : ainsi, le débat eut lieu à Orléans autour de la prise en compte de l'intérêt architectural et historique des faubourgs que la municipalité ne souhaitait pas inclure, au départ, dans le périmètre (l'architecte conseil de préciser que la commande politique ne concernait que l'intra-mail). C'est donc bien la culture du consensus qui est mise à l'honneur, un mode de faire ensemble. C'est une action publique ouverte, qui travaille en partenariat.

\section{CONCLUSION}

Si les procédures contractuelles se développent dans le contexte français depuis les lois de décentralisation, il ne faut cependant pas oublier que l'Etat reste le maître des jeux : "la permanence du pouvoir d'Etat correspond à un impératif juridique et à une nécessité pratique (MAILLOT, 1993). Mais de ce fait, la véritable gouvernance au sens de celle qui permet une régulation de l'action publique sous couvert de partenariat et de négociation, reste un fantasme largement cité mais peu utilisé concrètement. Si des processus de négociation de l'action patrimoniale ont bien eu lieu à Orléans lors de l'élaboration de la ZPPAUP, ce n'est que parce que les rapports entre la collectivité et les services de l'Etat étaient acceptables et permettaient une mise en débat de cette action. Toutefois, l'aval de l'Etat est nécessaire dans la procédure. Celui de la commune l'est aussi, c'est vrai, mais au final, c'est bien l'implication de l'Etat (via l'action des architectes des bâtiments de France) qui est négociée, en aucun cas son éradication du champ patrimonial local. La gouvernance patrimoniale semble se profiler à l'horizon mais reste, à mon sens, enchaînée par des jeux de pouvoirs qui donnent la part belle à l'Etat, garant de la ressource patrimoniale au nom de sa légitimité. Si les ZPPAUP sont bien des documents décentralisés, puisqu'elles permettent à la collectivité territoriale d'entrer sur la scène de débat, elles ne donnent pas à celle-ci une autonomie complète, mais définissent une politique patrimoniale explicite. D’une régulation patrimoniale en cercle fermé, l'on bascule sur un mode de régulation lisible et clairement visible par tous les acteurs du champ patrimonial d'une part mais aussi par les citoyens d'autre part. La gouvernance d'une action patrimoniale n'est pas un mythe, mais elle ne me semble pas complète et le terme peut être abusif dans ce domaine alors que celui de régulation paraît mieux convenir aux types de relations qui se (re)définissent entre les acteurs publics. La décentralisation de l'action patrimoniale a connu en France une avancée sans précédent avec les ZPPAUP mais l'on sent bien que le 
système étatique traditionnel dans ce domaine n'est pas encore prêt à s'effacer plus fortement.

\section{REFERENCES}

Baron C. (2003). La gouvernance : débats autour d'un concept polysémique. Droit et société, $54,329-351$

Choay F. (1996). L'allégorie du patrimoine. Paris : Seuil

Delaunay B. (1995). Les zones de protection du patrimoine architectural, urbain et paysager. Annuaire des collectivités locales, 487-501

Le Galès P. (1998). Régulation, gouvernance et territoire. In Commaille J. \& Jobert B. (Dir.) Les métamorphoses de la régulation politique (pp. 203-240). Paris : LGDJ

Gaudin J.P. (1995). Politiques urbaines et négociations territoriales. Revue française de science politique, $45,31-56$

Gigot M. (2007). Patrimoine et instruments d'action publique: le cas de la ZPPAUP d'Orléans, Tours : Université François-Rabelais

Grésillon M. (1995). Orléans. Paris : Anthropos

Hernandez F. (2003). Le processus de planification des déplacements urbains : entre projets techniques et modèles de ville. Aix en Provence : Université d'Aix-Marseille III

Lorrain D. (1998). Administrer, gouverner, réguler. Les annales de la recherche urbaine, 80$81,85-92$

Maillot D. (1993). Dix ans d'urbanisme décentralisé. Réflexions à l'occasion d'un anniversaire. Etudes foncières, 59, 34-40

Mirieu De Labarre E. (2004). Plaidoyer pour un nouveau régime des abords. In Audrerie D. \& Prieur M. (Dir.) Les monuments historiques, un nouvel enjeu? (pp. 11-62). Paris: l'Harmattan

Morand-Deviller J. (1985). Les procédures spécifiques de protection du patrimoine culturel. In Jegouzo Y. Droit du patrimoine culturel immobilier (pp. 67-80). Paris : Economica

Tomas, F. (2004). Les temporalités du patrimoine et de l'aménagement urbain. Géocarrefour, $79,197-211$

Woll, C. (2004). Régulation. In Boussaguet L., Jacquot S. \& Ravinet P. (Dir.) Dictionnaire des politiques publiques (pp. 377-384). Paris : Presses de Sciences Po 